\title{
Conceptual framework for sewer pump problems allowing for fuzzy logic application
}

\author{
AJ Bester ${ }^{1}$, HE Jacobs ${ }^{1 *}$ and J Tulleken ${ }^{1}$ \\ 'Department of Civil Engineering, Stellenbosch University, Private Bag X1, Matieland, 7602, South Africa
}

\begin{abstract}
Sewer pump station problems in separate sewer systems (separate from stormwater drainage systems) were investigated in order to derive a conceptual framework for decision making. Notwithstanding the fundamental service that efficient sewage pumping provides to a community, it was apparent that limited research has been published on the topic. Research was undertaken to identify, investigate and classify problems at existing operational sewage pump stations by means of site visits, interviews, and an extensive knowledge review. A conceptual framework for sewer pump problems was subsequently derived. The application of the framework is illustrated by means of a fuzzy logic approach to one of the problem classes identified. This study sets the scene for further research into fuzzy-based efficiency indices pertaining to different sewer system components and the ultimate application in sewer system decision support tools.
\end{abstract}

Keywords: sanitary sewers, pump station problems, framework

\section{INTRODUCTION}

The need for pumping sewage arises frequently in order to ultimately deliver the sewage flow to the waste water treatment works (WWTW). Steel (1960) lists a few specific conditions where the pumping of sewage is necessary, including provision of drainage for basements of buildings that are lower than the connection to the common sewer system and where the sewer system outlet at any collection point is below the receiving system. Instances where the topography would result in very deep excavations in the light of the required gravity pipe gradients may necessitate a sewer pump station to optimise the system cost. The focus of this paper is on sewer pump stations and associated problems in separate sewer systems.

This is the first publication pertaining to sewer pumps presented in Water SA since the inception of the journal in 1975. This paper adopts terminology used by Jacobs et al. (2011), Van Vuuren and Van Dijk (2011a) and Van Vuuren and Van Dijk (2011b).

It is impossible to design an entirely fool-proof system. In addition, unpredictable external influences, such as power failures, mechanical failures and system abuse, will always be present. These undesired and unexpected events lead to problems at sewer pump stations that require operational and maintenance inputs in order to resolve. Ultimately it would be useful to develop a decision support tool to link the design aspects to particular problems noted in the field. However, before such a link can be established it is necessary to identify and classify the most common problems, and to describe classes and individual problems in terms of a suitable framework, i.e. one that allows for further development and application. The development of such a framework, and illustration of its possible application, is the focus of this text.

\footnotetext{
* To whom all correspondence should be addressed.

ฮ +27 21808 4059; Fax: +27 21808 4351; e-mail: hejacobs@sun.ac.za Received 15 March 2012; accepted in revised form 9 March 2015
}

\section{RESEARCH PROBLEM}

\section{Classification of sewer pumps}

Pumps are classified in different ways, with detailed descriptions of different classification systems available in the literature (Jones et al., 2006). These classifications were critically reviewed in order to determine a link between pump categories and pump-problem categories; however, no clear link could be established. Two typical pump classification parameters are presented below. According to Fair et al. (1971), pumps could be classified as follows: centrifugal pumps, propeller pumps, displacement pumps, rotary pumps, hydraulic ram pumps, jet pumps, air lift pumps and displacement ejectors. An alternative classification system (Fraenkel, 1986) presents 5 major groups, each describing the method used for moving the fluid: direct lift, displacement, velocity, buoyancy and gravity pumps.

The focus of this study is on pumps used in the piped sewer system rather than at the WWTW. In this instance the most common pump type is a centrifugal pump, with various types of impellers typically used, such as vortex and screw centrifugal impellers. The mechanical efficiency of the pump impeller would normally decrease with increased solids handling ability, in terms of the solid item size to be passed.

The pumping station facility includes the pumps, equipment and structure necessary for pumping fluids. The pumping station thus includes the infrastructure surrounding the pump, such as the building, sumps, hoisting equipment, electrical and electronic equipment, solids handling devices (SHD), security fences and access roads.

\section{PUMP STATION PROBLEM OVERVIEW}

The following is a list of the most notable problems, based on interviews with various maintenance personnel and a review of maintenance management systems: clogging and unwanted solid objects; fats, oils and grease (FOG) deposits; peak flows (particularly referring to wet weather peak flows where there is considerable storm water ingress into the conveyance system); electrical power supply failure; lack of proper routine maintenance 
procedures; vandalism; inadequate design of the pump station.

During the problem identification phase it became apparent that some boundaries had to be put in place to prevent proliferation of problems. A 'problem' was subsequently limited to something that could be determined by means of inspection at the pump station site, the operation and maintenance office, or by reviewing design documentation. Various common problems have been documented and a comprehensive list of all reported and recorded problems were compiled during this study. The problem list was compiled from literature, site visits and stakeholder interviews. Some common problems pertaining to sewers and pumps were also listed by Van der Merwe-Botha and Manus (2011) and Sidwick (1984) and included in the list.

\section{Conceptual framework and problem classes}

The approach followed in this research was that the problem classes for the framework could be derived by starting small and successively adding complexity based on subjective judgement and stakeholder input. Knowledge obtained from the responsible operational staff and from site visits was continually added into the process, thus building towards a relatively robust framework that would incorporate all of the main types of problems.

The research team based the methodology of this study on successful frameworks derived elsewhere. The comprehensive European Union (EU) framework for flood disaster management is an example of a robust framework allowing for stakeholder participation, as well as defining roles and responsibilities. Ashley et al. (2011) reported that the EU framework, whilst retaining the processes of prevention, protection, preparedness, emergency response and recovery and lessons learned, provides a visualisation in which activities may be carried out in parallel processes, which may intertwine and merge or separate as appropriate. The EU framework links specific roles and responsibilities to each component of the framework. The Scottish Government's Four A's approach (Ashley et al., 2011) was incorporated into the EU framework and grouped as follows:

- Awareness - of flood risk management

- Analysis - and assessment of flood risk

- Alleviation - and avoidance of flooding

- Assistance - prior to, during and after flood events

Sewer pump problems were considered amenable to the same approach in that the A's listed above would, for example, provide the basis for a framework to address and resolve problems. The sewer pump station problem framework would thus incorporate implied knowledge as well as experience to make logical sense based on the quagmire of information. Tulleken (2012) categorised sewer pump station problems into four classes. The four classes were termed the four O's of sewage pump station problems. These four classes were: overflows, odours; operational (and maintenance); other. The aim of this investigation was to use the four O's as classification standard for the fuzzy sets (discussed below).

\section{CATEGORISING PROBLEMS}

\section{The concept and application of categorisation}

A detailed account of categorisation is beyond the scope of this text, but a brief review is considered necessary in order to clearly present the subsequent framework and fuzzy-based illustration.
Cohen and Lefebvre (2005) described categorisation as the process in which ideas and objects are recognised, differentiated and understood. Categorisation thus entails the grouping or sorting of certain objects into pre-defined categories for some specific purpose, based on logical relationships between these objects. Ideally, a category would illuminate the relationship between the subjects and objects of knowledge. Categorisation is fundamental in many fields of study, including decision making.

One of the major flaws made with categorisation is when objects are placed in a category where they do not belong. This is termed miscategorisation. This occurs when dissimilar objects are accidentally grouped together based upon common denominators (any object would have this variable in common), or illogical denominators.

Consider, for example, the simple case of a power failure, leading to an overflow and subsequent odour problem. What is the problem and how would it be categorised? Apart from the actual electricity failure this particular problem could correctly be described as any one of the following: insufficient emergency storage and thus insufficient pump sump size, the lack of backup power, odour problems (due to the spillage), or excess peak inflow during a rain event combined with any of the former.

As part of this research conceptual clustering was used to place the different entities (the sewer pump problems) into various classes by first formulating the conceptual description of each class and then classifying the entities according to the descriptions of each class. Conceptual clustering is related to fuzzy set theory, in which objects may belong to one or more groups (in this case classes), in varying degrees of fitness. This makes sense for the classification of sewer pump problems, where a given pump station problem could for example belong to more than one of the classes, but in varying degrees of membership in each case.

\section{Existing problem categories}

The City of Cape Town (Samson, 2011) listed 5 main risk categories associated with sewer pump stations. The five categories identified were: mechanical failure of duty pump sets, mechanical failure of duty and standby pump sets, electrical failure within the pump station, power outages, and blockages of the SHD.

During site visits and discussions with operational personnel, maintenance management systems were found to present lists of problems, and in some instances there was some form of categorisation. However, based on the nature of the work the systems focused on, e.g., mechanical and electrical problems only, or often only on pump problems, in contrast to pump station problems (which may include, e.g., vandalism, theft, or access problems to the site). For this reason a broader framework encompassing a wider range of pump station problems was developed during this research project.

\section{Quantifying problems}

Identifying, listing and classifying the problems was a particular challenge faced by the research team, as well as the procedure for quantifying the problems. One possible method was by means of assessing the reliable operation, in other words, determining the system reliability. Reliability is often determined as part of general engineering work and is site-specific. The outcome would 
thus not be generically applicable to other pump stations and is not as amenable to future optimisation as other methods. The term 'problem' is used loosely in this research project, despite the understanding that in a purely technical sense the definition could be linked to system reliability.

\section{FUZZY SET THEORY AND THE APPLICATION TO SEWER PUMP STATIONS}

A fuzzy concept is a concept for which the meaningful content, value, or boundaries of application can vary considerably according to context or conditions, instead of being fixed to scenarios (Haack, 1996). This generally means the concept is vague, lacking a fixed, precise meaning, but without, however, being meaningless altogether (Dietz and Moruzzi, 2009). Fuzzy control brings essential advantages, such as the use of algorithms in the case of defining affirmations by logical expressions. Over the past several years, fuzzy control has emerged as one of the most active and fruitful research areas in the applications of fuzzy set theory, especially in the realm of industrial processes, which do not lend themselves to control by conventional methods because of a lack of quantitative data regarding the input-output relations (Mariana, 2011). A few examples of fuzzy logic application to water services are presented in Table 1.

\section{Crisp weighed ranking}

Crisp weighed ranking is based on the principles of classical set theory, the basis for most decision making processes. Classical set theory allows for two options: either something is a member of a set or it is not a member. An example of a classical or crisp set, as explained by Hardy (1994) would be the set of mammals. In this set a dog would be a member of the set whereas an eagle is not. Difficulties arise, however, when the platypus is considered in the set of mammals (this example will further be discussed in the following section), because it could be placed in more than one set.

\section{Fuzzy scheme for ranking}

The platypus lays eggs and has a duck-like bill, yet is covered with fur. According to classical set theory the platypus must either be a member of the set of mammals or not a member. In fact, the platypus is somewhat a mammal and somewhat not a mammal, so it is a partial member of this set. Fuzzy logic accommodates such cases through partial membership (Brubaker, 1992).

Often in decision-making environments we are faced with the problem of selecting one from among a collection of alternative actions. This selection process may then require that options are ranked, or ordered. In many applications of fuzzy set theory it is necessary to obtain a measure of a course of action expressed as a fuzzy number. Essentially there is a level of uncertainty as to the exact value of the entity. As noted by Kosko (1993) this is a kind of possibilistic uncertainty. While it is clear when considering two pure numbers which is bigger or smaller the situation with respect to fuzzy numbers is not always obvious.

\section{Method for comparing fuzzy numbers}

The general approach when comparing fuzzy numbers is to associate with a fuzzy number $F$ some representative single value, $\operatorname{Val}(F)$, and compare the fuzzy subsets using single representative values. Yager (1981) mathematically expressed this approach of obtaining a unique value as the proxy of a fuzzy subset using:

$$
\operatorname{Val}(F)=\int_{0}^{1} \operatorname{Ave}\left(F_{x}\right) d x
$$

In this case $F_{x}=\{x \mid F(x) \geq x\}$ is the $x$-level set and Ave $\left(F_{\mathrm{x}}\right)$ is the average of the elements in the $x$-level set. In essence $\operatorname{Val}(F)$ can be considered an averaging operation.

\begin{tabular}{|l|l|l|}
\hline \multicolumn{2}{|l}{ Chronological review of related fuzzy logic applications } \\
\hline Reference & \\
\hline 1992 & Hou and Ricker (1992) & Minimised combined sewer overflows (CSO) in a 3-reservoir system \\
\hline 1993 & Hirota (1993) & $\begin{array}{l}\text { Programmable controllers used to develop the so called FRUITAX system - applied to water } \\
\text { treatment and rain water pumping processes }\end{array}$ \\
\hline 1995 & Bardossy and Duckstein (1995) & Fuzzy adaptive system (FAS) used to determine the control problem of reservoir operations \\
\hline 1996 & Tsai et al. (1996) & $\begin{array}{l}\text { Controlled suspended solid concentration through the implementation of an on-line fuzzy logic } \\
\text { controller }\end{array}$ \\
\hline 1997 & Iwanaga et al. (1997) & $\begin{array}{l}\text { Comparative study on the use of fuzzy logic and multiple regression analysis for chemical water } \\
\text { quality analysis and taste tests }\end{array}$ \\
\hline 1997 & Panigrahi and Mujumdar (1997) & $\begin{array}{l}\text { Applied the concept of a fuzzy rule-based system to determine the releases from a large-scale } \\
\text { irrigation reservoir }\end{array}$ \\
\hline 1999 & Kalker et al. (1999) & Determined optimal dosages of chemicals in a wastewater treatment (activated sludge) process \\
\hline 1999 & Pongracz et al. (1999) & Forecast of droughts in a region of North America \\
\hline 1999 & Dou et al. (1999) & FAS applied for solute transport simulation \\
\hline 2000 & Abebe et al. (2000) & FAS used for filling gaps of incomplete precipitation data \\
\hline 2011 & $\begin{array}{l}\text { Ostojin et al. (2011) } \\
\text { inger }\end{array}$ & $\begin{array}{l}\text { Applied fuzzy logic to optimise energy costs at a sewage pump station by modifying the on-off } \\
\text { switching rules for pumps. The work pertains to combined storm sewers. A genetic algorithm } \\
\text { search technique was derived to adjust the parameters that define the membership functions in } \\
\text { the fuzzy rules, in order to minimise energy costs towards an optimal solution. From their work } \\
\text { a thorough foundation and solid framework could be established, leading to the application of } \\
\text { advanced techniques in terms of improved decision support and optimisation. }\end{array}$ \\
\hline
\end{tabular}




\section{Fuzzy sets}

Fuzzy sets are described domains of the inputs, each of which is thought to have a definite effect on the output. The system input parameters are defined by the analyst. As an example, Ostojin et al. (2011) combined the rate of level change in the pump sump and the actual level in the sump as inputs. Any number of inputs could be used. In developing an automated control system for sewage pump stations in Taipei City, Chiang et al. (2010) used input parameters for water level, precipitation, status of pumps, status of sluice gates and the predictive water level as a basis for their fuzzy sets.

The inputs for the case of sewer pump problems are the pump station problem entities, each classified and thus belonging in some degree of membership to conceptual classes, namely the four O's. Each of these classes is sub-divided into a number of sub-classes as discussed earlier, and thus comprises different inputs depending on the particular sub-set evaluated.

Referring again to the example presented earlier: the simple case of a power failure leading to an overflow and subsequent odour problem. It is now possible to identify as an output some type of rank to determine what problem would be the most 'notable' in terms of the rule space. This would not tell the analyst how to solve the problem, but would aid in identifying the problem that is expected to be the 'worst' as defined by the analyst setting up the rule space. The values of system inputs are defined by a degree of membership, with membership values given in integers ranging from 1 to 5 in this case. The framework of the four O's allows for robust yet flexible application of fuzzy set theory.

\section{Fuzzy logic controller structure}

In the application to sewer pump stations, general fuzzy controller architecture is proposed, as shown in Fig. 1.

A fuzzy logic controller comprises 4 main components (Navarro et al., 1990):

- Fuzzification interface (FI) - measures the values of the input values and performs a scale mapping that transfers

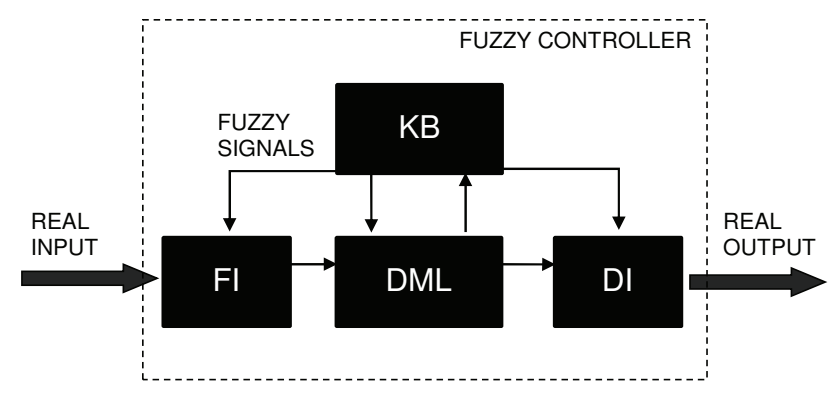

Figure 1

Fuzzy logic controller structure the range of values of input variables into corresponding spaces of discourse

- Knowledge base (KB) - provides necessary definitions, which are used to define control rules and fuzzy data manipulation in a fuzzy logic controller

- Decision-making logic (DML) - capable of simulating human decision-making based on fuzzy concepts and of inferring fuzzy control actions employing fuzzy implication and the rules of inference in fuzzy logic

- Defuzzification interface (DI) - performs a scale mapping, which converts the range of values of output variables into corresponding spaces of discourse, by yielding a non-fuzzy control action from an inferred fuzzy control action

In summary: fuzzy set theory comprise 3 components, namely, fuzzy sets representing the real system inputs, the rule space that is typically presented as a set of IF-THEN statements (fuzzy controller) and the real system outputs. A fuzzy system develops rules to match any set of input-output data and develop useful decision support systems.

\section{ILLUSTRATION OF APPLICATION}

This section illustrates the possible future application (in an overly simplified manner) of the framework in further developing a decision support tool pertaining to sewer pump station problems. An overview on sub-set values attributed to each scenario pertaining to one of the four O's, namely, 'overflows', is presented first to illustrate the application.

\section{Weights and sub-set values for class 'overflows'}

The application of fuzzy logic is demonstrated as it pertains to the class for overflows. Plugh's method comprises a basic decision matrix to compare a set of statements, or problem entities in this case, in terms of some criteria by allocating a weight to each based on subjective decisions (Ullman, 1992). Each entity is given a weight in this manner by comparing it to other entities in the same sub-set. Each is weighed against each other by allocating a score; in this example, between 1 and 10 in each case. The criterion with 'higher importance' based on the subjective series of decisions thus receives the higher score and subsequent higher ranking. The problem entity with the highest weight receives the highest rank representing the degree of membership.

The class for 'overflows' comprises 6 sub-classes, namely: blockages, mechanical failures, electrical failures, peak flows, power outages and storage failure. Each of these comprises a set of problem entities. These individual problem entities are scored against each other using Plugh's method, as demonstrated in the following tables.

\begin{tabular}{|l|c|c|c|c|}
\hline \multicolumn{7}{|c|}{ Table 2 } \\
& Evaluating entity weights for blockages \\
\hline SHD impassable & Unwanted objects & FOG deposits & Grit accumulation \\
\hline Unwanted objects & $*$ & 7 & 6 & 5 \\
\hline FOG deposits & 3 & $*$ & 4 & 4 \\
\hline Grit accumulation & 4 & 6 & $*$ & 6 \\
\hline Weight & 5 & 6 & 4 & * \\
\hline Rank & $\mathbf{1 2}$ & $\mathbf{1 9}$ & $\mathbf{2}$ & $\mathbf{1 4}$ \\
\hline
\end{tabular}


Table 3

Evaluating entity weights for mechanical failures

\begin{tabular}{|c|c|c|c|c|c|}
\hline & $\begin{array}{c}\text { Design } \\
\text { deficiencies }\end{array}$ & $\begin{array}{c}\text { Unwanted } \\
\text { objects }\end{array}$ & Parts failure & Wear and tear & $\begin{array}{l}\text { Lifetime } \\
\text { complete }\end{array}$ \\
\hline Design deficiencies & * & 7 & 6 & 4 & 4 \\
\hline Unwanted objects & 3 & * & 5 & 4 & 3 \\
\hline Parts failure & 4 & 5 & * & 4 & 4 \\
\hline Wear and tear & 6 & 6 & 6 & * & 6 \\
\hline Lifetime complete & 6 & 7 & 6 & 4 & * \\
\hline Weight & 19 & 25 & 23 & 16 & 17 \\
\hline Rank & 3 & 5 & 4 & 1 & 2 \\
\hline
\end{tabular}

Table 4

Evaluating entity weights for electrical failures

\begin{tabular}{|c|c|c|c|c|}
\hline & Wiring & Level probes failure & $\begin{array}{c}\text { Failure of alarm, } \\
\text { telemetry or } \\
\text { monitoring } \\
\text { equipment }\end{array}$ & Switching failure \\
\hline Wiring & * & 4 & 3 & 4 \\
\hline Level probes failure & 6 & * & 4 & 5 \\
\hline $\begin{array}{l}\text { Failure of alarm, telemetry or moni- } \\
\text { toring equipment }\end{array}$ & 7 & 6 & * & 7 \\
\hline Switching failure & 6 & 5 & 3 & * \\
\hline Weight & 19 & 15 & 10 & 16 \\
\hline Rank & 4 & 2 & 1 & 3 \\
\hline
\end{tabular}

Table 5

Evaluating entity weights for peak flows

\begin{tabular}{|l|c|c|c|c|}
\hline & Vacation period & Stormwater ingress & Illegal linkage & $\begin{array}{c}\text { Swimming pool } \\
\text { overflows }\end{array}$ \\
\hline Vacation period & $*$ & 6 & 5 & 3 \\
\hline Stormwater ingress & 4 & $*$ & 4 & 3 \\
\hline Illegal linkage & 5 & 6 & $*$ & 4 \\
\hline Swimming pool overflows & 7 & 7 & $\mathbf{1 5}$ & $*$ \\
\hline Weight & $\mathbf{1 6}$ & $\mathbf{1 9}$ & $\mathbf{2}$ & $\mathbf{1 0}$ \\
\hline Rank & $\mathbf{3}$ & $\mathbf{4}$ & $\mathbf{1}$ \\
\hline
\end{tabular}

Table 6

Evaluating entity weights for electrical power outages

\begin{tabular}{|l|c|c|c|c|}
\hline & Supply failure & Generator failure & Cable theft & Switching failure \\
\hline Supply failure & $*$ & 6 & 4 \\
\hline Generator failure & 4 & * & 4 \\
\hline Cable theft & 4 & 7 & 4 \\
\hline Switching failure & 6 & $\mathbf{1 9}$ & 6 \\
\hline Weight & $\mathbf{1 4}$ & $\mathbf{4}$ & $\mathbf{1 6}$ \\
\hline Rank & $\mathbf{2}$ & $\mathbf{1 1}$ & $\mathbf{3}$ \\
\hline
\end{tabular}




\begin{tabular}{|l|c|c|c|c|}
\hline \multicolumn{5}{|c|}{ Evaluating entity weights for storage failure } \\
\hline & Inflow>outflow & $\begin{array}{c}\text { Insufficient } \\
\text { emergency sump } \\
\text { storage }\end{array}$ & Structural failure & $\begin{array}{c}\text { Inadequate } \\
\text { overflow facilities }\end{array}$ \\
\hline Inflow>outflow & $*$ & 4 & 4 & 4 \\
\hline Insufficient emergency sump storage & 6 & $*$ & 4 & 4 \\
\hline Structural failure & 6 & 6 & $*$ & 6 \\
\hline Inadequate overflow facilities & 6 & 6 & 4 & $\mathbf{1 2}$ \\
\hline Weight & $\mathbf{1 8}$ & $\mathbf{1 6}$ & $\mathbf{1}$ & $\mathbf{2}$ \\
\hline Rank & $\mathbf{4}$ & $\mathbf{3}$ & & 2 \\
\hline
\end{tabular}

\begin{tabular}{|l|c|c|c|c|c|c|}
\hline \multicolumn{7}{|c|}{ Degree of membership for entities per sub-class of 'overflows' } \\
\hline & Blockages & Mechanical & Electrical & Peak flows & Power outages & Storage failure \\
\hline Blockages & $*$ & 3 & 3 & 4 & 4 & 5 \\
\hline Mechanical & 7 & $*$ & 5 & 6 & 6 & 7 \\
\hline Electrical & 7 & 5 & $*$ & 6 & 5 & 5 \\
\hline Peak flows & 6 & 4 & 4 & $*$ & 4 & 6 \\
\hline Power outages & 6 & 4 & 5 & 6 & $*$ & 6 \\
\hline Storage failure & 5 & 3 & 5 & 4 & 4 & $*$ \\
\hline Weight & $\mathbf{3 1}$ & $\mathbf{1 9}$ & $\mathbf{2 2}$ & $\mathbf{2 6}$ & $\mathbf{2 3}$ & $\mathbf{2}$ \\
\hline Rank & $\mathbf{6}$ & $\mathbf{1}$ & $\mathbf{2}$ & $\mathbf{4}$ & \multicolumn{3}{|c|}{$\mathbf{3}$} \\
\hline
\end{tabular}

The scoring of weights in Table 2 to Table 7 is based on a relatively small pump station that was considered a median of those visited during this research project in the Western Cape, South Africa.

Subjective scores are allocated to each entity in comparison to the rest. This would typically be done in collaboration with technical and maintenance staff. This decisionbased procedure would be site specific.

The rank was subsequently obtained from the weight in Table 2. The values listed below describe the degree of membership of each entity as an integer, used in applying the fuzzy logic rules (Rank 1 is least significant and Rank 4 most significant):

- Unwanted objects $=4$

- Grit accumulation $=3$

- FOG deposits = 2

- $\quad$ SHD impassable $=1$

The entity with the lowest weight would be the one that was considered to be the least significant in terms of its impact (compared to the others in this sub-set), and therefore would receive the lowest score. This technique was applied to all of the other sub-classes. The evaluation of entity weights for the remainder of the subsets is presented in Tables 3 to 7 .

The ranked value of each entity provides a measure of the degree of membership. It would be possible, of course, to assess the weights in a different manner allowing for more complex decision methods.

The method was also extended to distinguish between the six sub-classes of the class 'overflows'. These sub-classes were subsequently weighed against each other in the same manner as before and the result is presented in Table 8, with a rank for each of the subclasses.

\section{Application of technique}

Refer to Table 8 and consider an unwanted object arriving in the sewage stream at the pump station. It might lead to blockages (Table 2) or mechanical failures (Table 3). The significance can now be ranked and compared. Fuzzy sets describe the degree of membership for each entity (rank derived from Table 2 to Table 7) and this membership was multiplied with the degree of membership of the sub classes (rank derived from Table 8), giving each entity in the class overflows a value. This method is explained with the 'IF' statements as indicated below:

- IF (blockage due to unwanted object) THEN (6 x 4) = 24

- IF (mechanical failure due to unwanted object) THEN

$$
(1 \times 5)=5
$$

The unwanted object causing a blockage is approximately 5 times more significant than it causing a mechanical failure. This makes sense, because if a mechanical failure were to occur a back-up pump would take over, or the sump level alarm would trigger a standby mobile pump unit to be employed in a relatively short period of time. However if a blockage were to occur at the SHD at the inlet to the pump station, an overflow could result in a relatively short period of time, without warning. A similar method of applying fuzzy logic could be applied to all four O's of pump station problems in combination.

\section{DISCUSSION}

The framework for sewer pump station problems could be a basis from which to derive more advanced decision support tools pertaining to sewer pump problems. The concept of fuzzy logic should be extended to all four O's and the degree 
of membership should allow for individual entities to share membership between different classes and sub-classes. This extension would necessarily entail the development of specific software.

Presuming that pump problems could be optimally ranked in this way by an advanced decision support system, ultimately each problem still needs to be addressed by a responsible person. The derived framework would ideally also aid future communication between different staffing levels (or stakeholders) in terms of pump station problems. Blanksby et al. (2011) noted that, by focusing on tasks and roles in framework development, the different stakeholders are able to cut through major barriers to communication. These aspects could be incorporated into the framework by identifying specific tasks and roles pertaining to each problem entity, sub-class and class. Individual roles and responsibilities could be delineated, which in turn would minimise or mitigate pump station problems in the long run.

The application of genetic algorithms to optimise fuzzy sets for sewer pumps at a single pump station was achieved by Ostojin et al. (2011). Their work shows that a thorough foundation and fuzzy-based framework could lead to the application of advanced techniques in terms of decision support and ultimately optimisation.

\section{CONCLUSION}

This paper presents a novel framework for sewage pump station problems in separate sewer systems. As part of this research it was necessary to identify and classify the most common problems, and to describe classes and individual problems in terms of a suitable framework - one that allows for further development and application.

The framework for problems at sewage pump stations incorporates four problem classes, termed the four O's of sewer pump station problems: overflows, odours, operational and other. The typical application of the framework by means of a fuzzy logic approach was illustrated.

\section{ACKNOWLEDGEMENTS}

The South African Water Research Commission (WRC) is greatly acknowledged for their significant contribution to this work in terms of a 3-year funding grant and guidance provided by the project reference group. The authors would like to acknowledge the various local authorities and their staff who made information available as part of the WRC project, and the collaborators AECOM and University of Johannesburg. Also, credit is due to former researchers for publishing results and in so doing providing others with a scientific foundation to work from.

\section{REFERENCES}

ABEBE AJ, SOLOMATINE DP and VENNEKER RG (2000) Application of adaptive fuzzy rule-based models for reconstruction of missing precipitation events. Hydrol. Sci. J. 45 (3) 425-436.

ASHLEY RM, BERTRAND-KRAJEWSKI JL, HEVITED-JACOBSON T and VERBRANCK M (2004) Solids in Sewers: Characteristics, Effects and Control of Sewer Solids and Associated Pollutants. IWA Publishing, London. 340 pp.

ASHLEY R, BLANKSBY J, MAGUIRE T and LEAHY T (2011) Frameworks for adapting to floodrisk: experiences from the EU's flood resilient city project. In: Proceedings of the 1st IAHR European Congress, 4-6 May 2011, Sheffield.
BARDOSSY A and DUCKSTEIN L (1995) Fuzzy Rule-Based Modelling with Applications in Geophysical, Biological and Engineering Systems. CRC Press, Boca Raton, Florida. 256 pp.

BLANKSBY J, ASHLEY R and WALKER L (2011) The development of a planning framework for flood risk and water management at city or country scale. In: Proceedings of the 12th Annual Conference on Urban Drainage, 1 May 2011, Porto Alegre.

BRUBAKER DI (1992) Fuzzy logic basis: intuitive rules replace complex math. EDN Design Feature 37 (13) 111-116.

CHIANG YM, CHANG LC, TSAI MJ and CHANG FJ (2010) Autocontrol of pumping operations in sewerage systems by rule-based fuzzy neural networks. Hydrol. Earth Sci. 15 (1) 185-196.

COHEN H and LEFEBRE C (2005) Handbook of Categorization in Cognitive Science. Elsevier, London. $1081 \mathrm{pp}$.

DIETZ R and MORUZZI S (2009) Cuts and Clouds: Vagueness, its Nature, and its Logic. Oxford University Press, Oxford. 575 pp.

DOU C, WOLDT W and BOGARDI I (1999) Fuzzy rule based approach to describe solute transport in the unsaturated zone. J. Hydrol. 220 (2) 74-85.

HAACK S (1996) Deviant Logic, Fuzzy Logic: Beyond the Formalism. University of Chicago Press, Chicago. 318 pp.

HARDY TL (1994) Fuzzy logic approaches in multi-objective decisionmaking in aerospace applications. In: Proceedings of the 30th Joint Propulsion Conference, 27-29 June 1994, Colorado Springs.

HIROTA K (1993) Industrial Applications of Fuzzy Technology. Springer-Verlag, Tokyo. 310 pp.

HOU SL and RICKER NL (1992) Minimization of combined sewer overflows with fuzzy logic control. In: Proceedings of the IEEE International Conference on Fuzzy Systems, 8-12 March 1992, San Diego.

IWANAGA C, SASAKI K and HAMOAKA T (1997) Evaluation of the quality of water using fuzzy reasoning and multiple regression analysis. Jap. J. Fuzzy Theory Syst. 9 (3) 291-306.

JACOBS HE, FAIR K, DE KLERK AH and BESTER AJ (2011) Sewer system planning made simple - for small local authorities. WRC Report No. 1828/1/11. Water Research Commission, Pretoria. $166 \mathrm{pp}$.

JONES GM (2006) Pumping Station Design. Elserivier, Amsterdam. 1104 pp.

KALKER T, BABUSKA R, ROELEVELD P, RULAND M and VAN GOOR C (1999) Toepasbaarheid van fuzzy control bij het zuiveren van stedelijk afvalwater. $H 2 O 1$ (1) 14-17.

KOSKO B (1993) Fuzzy Thinking: The New Science of Fuzzy Logic. Hyperion, New York. 318 pp.

MARIANA HT (2011) Application of fuzzy set theory in controlling of urban storm water drainage systems. PhD thesis, Gh.Asachi Technical University of Iaşi, Romania.

NAVARRO JL, ALBERTOS P, MARTINEZ M and MORANT F (1990) Intelligent industrial control. In: Proceedings of the 11th IFAC Triennial World Congress, 13-17 August 1990, Tallin.

OSTOJIN S, MOUNCE SR and BOXALL JB (2011) An artificial intelligence approach for optimizing pumping in sewer systems. J. Hydroinf. 13 (3) 295-306.

PANIGRAHI DP and MUJUMDAR PP (1997) Application of fuzzy logic to reservoir operation modelling. In: Proceedings of National Symposium on Fuzzy Sets and their Applications, 9-10 June 1997, Madras.

PONGRACZ R, BOGARD I and DUCKSTEIN L (1999) Drought forecasting using atmospheric circulation and ENSO information. In: Proceedings of 28th IAHR Congress, 22-27 August 1999, Graz.

SAMSON K (2011) Waste risk abatement plan of City of Cape Town. WRC Report No. SP 22/11. Water Research Commission, Pretoria. 27 pp.

SIDWICK JM (1984) Screening and Grid in Sewage - Removal, Treatment and Disposal. Construction Industry Research and Information Association, London. 83 pp.

STEEL EW (1960) Water Supply and Sewerage. McGraw-Hill, New York. 655 pp.

TSAI YP, OUYANG CF, WU MY and CHIANG WL (1996) Effluent suspended solid control of activated sludge process by fuzzy control approach. J. Water Environ. Res. 1 (1) 1045-1053.

TULLEKEN J (2012) Enquiry into sewage pump problems with the specific focus on removing solids. MSc(Eng) thesis, Stellenbosch University. 
ULLMAN DG (1992) The Mechanical Design Process. McGraw-Hill, New York. 337 pp.

VAN DER MERWE-BOTHA M and MANUS L (2011) Wastewater risk abatement plan - a W2RAP guideline. WRC Report No. TT 489/11. Water Research Commission, Pretoria. 108 pp.

VAN VUUREN SJ and VAN DIJK M (2011a) Waterborne sanitation design guide. WRC Report No. 11/40/01/10. Water Research Commission, Pretoria. $265 \mathrm{pp}$.
VAN VUUREN SJ and VAN DIJK M (2011b) Waterborne sanitation operation and maintenance guide. WRC Report No. 1144/02/10. Water Research Commission, Pretoria. 103 pp.

YAGER RR (1981) A procedure for ordering fuzzy subsets of the unit interval. Inf. Sci. 24 (1) 143-161.

\section{APPENDIX}

\section{List of acronyms}

CSO: $\quad$ Combined sewer overflows

FAS: $\quad$ Fuzzy adaptive system

FOG: $\quad$ Fats, oils and grease

SHD: $\quad$ Solids handling device

WWTW: Waste water treatment works 